\title{
Acht Jahre Deutschlandstipendium
}

\author{
Zur Entwicklung des Nationalen Stipendienprogrammes
}

Seit dem Jahr 2011 werden talentierte und engagierte Studienende in ganz Deutschland durch das Deutschlandstipendium gefördert. Dieses Förderinstrument hat sich fest in der Hochschulwelt etabliert und einen Beitrag dazu geleistet, an zahlreichen Hochschulen eine neue Stipendienkultur und Netzwerkstrukturen mit dem gesellschaftlichen und wirtschaftlichen Umfeld zu etablieren: Mittlerweile vergeben die über 300 teilnehmenden Hochschulen in eigener Verantwortung jährlich knapp 26.000 Stipendien an Studierende aller Fachrichtungen.

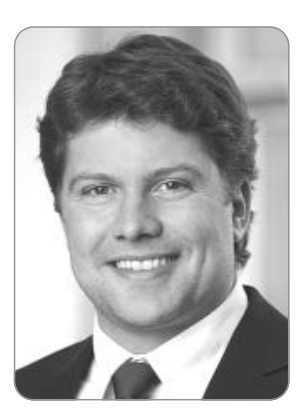

Dr. Alexander Tiefenbacher ist Projektleiter des Servicezentrums Deutschlandstipendium im Stifterverband für die Deutsche Wissenschaft.

\section{Stichwörter: Nachwuchsförderung, Stipendienver- gabe, öffentlich-private Partnerschaften, gesell- schaftliches Engagement}

\section{Entwicklung und Charakteristika}

Vor acht Jahren ist das Deutschlandstipendium mit der Absicht gestartet, in Deutschland eine neue Stipendienkultur zu begründen und den Misstand zu bekämpfen, dass damals nur rund ein Prozent aller Studierenden in Deutschland eine staatliche Stipendienförderung erhalten haben. Auch vor dem Hintergrund des drohenden Mangels von gut ausgebildeten Fachkräften erschien es geboten, dieses Defizit durch die Bereitstellung weiterer Anreize zur Aufnahme und erfolgreichen Durchführung eines Studiums aktiv zu bekämpfen.

Im Gründungsjahr 2011 konnten 5.400 Studierende durch ein Deutschlandstipendium gefördert werden. Über die Jahre hinweg ist die Anzahl der geförderten Studierenden kontinuierlich gestiegen: Im Jahr 2014 waren es bereits über 20.000 Stipendiaten und Stipendiatinnen und in 2017 knapp 26.000 Studierende. Neben den Begabtenförderungswerken, welche zusammen genommen eine ähnlich hohe Anzahl an Studierenden fördern, hat sich das Deutschlandstipendium damit als feste Größe der Talentförderung etabliert. Im Unterschied zu den Begabtenförderungswerken wird das Deutschlandstipendium dezentral von den einzelnen Hochschulen gestaltet, die bei der Umsetzung eigene Schwerpunkte setzen können, beispielsweise bei der Förderung von Studierenden aus nicht-akademischen Elternhäusern oder von Zuwanderern.

Auch die Art der Finanzierung stellt beim Deutschlandstipendium eine Besonderheit dar, da die vergebenen Stipendien gemeinsam aus öffentlichen und privaten Mitteln finanziert werden. Die Stipendiaten erhalten eine einkommensunabhängige Förderung von 300 Euro im Monat, von denen 150 Euro von einem privaten Förderer stammen und die anderen 150 Euro aus Bundesmitteln. Für die Stipendiaten sind diese Mittel weder steuer- noch sozialabgabenpflichtig und sie können sie, sofern sie bei den jährlichen Leistungskontrollen erfolgreich abschneiden, bis zum Ende der Regelstudienzeit ihres Bachelor- oder Masterstudiengangs erhalten. Wer schon eine begabungs- und leistungsabhängige materielle Förderung erhält, die durchschnittlich 30 Euro oder mehr pro Monat beträgt, kann kein Deutschlandstipendium mehr bekommen. Auf diese Weise soll sichergestellt werden, dass möglichst viele Studierende von einer finanziellen Studienunterstützung profitieren können. Stipendiatinnen und Stipendiaten der Begabtenförderungswerke beispielsweise müssen auf die materielle Förderung verzichten, wenn sie ein Deutschlandstipendium erhalten möchten. 


\section{Aufbau von langfristigen Netzwerkstrukturen}

Das zentrale Element dieses Förderprogrammes besteht in der Vernetzung der Hochschulen mit ihrem wirtschaftlichen und gesellschaftlichen Umfeld, also beispielsweise mit Unternehmen, Stiftungen, Vereinen und Privatpersonen (insbesondere Alumni) aus der jeweiligen Region. Im Jahr 2017 konnten durch den Aufbau derartiger Netzwerkstrukturen über 7.000 private Förderer gewonnen und 27 Millionen Euro aus privaten Quellen zur Stipendienvergabe bereitgestellt werden. Insgesamt konnten die Hochschulen seit dem Start des Programms rund 140 Millionen Euro private Mittel für das Deutschlandstipendium mobilisieren.

Träger und Treiber der Netzwerke sind dabei so unterschiedliche Knotenpunkte wie das Bundesministerium für Bildung und Forschung (BMBF), die einzelnen Hochschulen, die Stipendiaten sowie die privaten Förderer. Das Gros der operativen Vernetzungsarbeit kommt dabei zweifellos den Hochschulen zu. Ihre Aufgabe ist es, private Förderer von den Vorzügen einer Förderung zu überzeugen, da nur dann ein Stipendium vergeben werden kann, wenn ein privater Förderer gewonnen wurde, der breit ist, den privaten Finanzierungsanteil von 150 Euro im Monat zu übernehmen. Diese Summe wird dann auch vom Bund in gleicher Höhe zur Stipendienfinanzierung bereitgestellt. Bei diesem sog. Matching-Funds-Modell handelt es sich um ein bewährtes Instrument im Fundraising bzw. beim Aufbau von Kooperations- und Netzwerkstrukturen, mit dem eine Hebelwirkung von privatem Engagement erzielt werden kann. Darüber hinaus wird das Thema der Nachwuchs- und Bildungsförderung so zu einem gesamtgesellschaftlichen Anliegen.

Eine zu Beginn des Jahres 2016 veröffentlichte Evaluation und Begleitforschung zum Deutschlandstipendium, welche unter anderem Auskunft über Fördererprofile und Erfolgsfaktoren beim Einwerben privater Fördermittel gab, hat gezeigt, dass für 32 Prozent der privaten Mittelgeber das Deutschlandstipendium der Auslöser war, erstmalig eine Kooperation mit einer Hochschule zu beginnen, beziehungsweise sich im Bereich der Nachwuchsförderung zu engagieren. Dies zeigt, dass das Deutschlandstipendium hier ungenutzte Potentiale heben kann und den Aufbau von Netzwerken zwischen der Hochschule und ihrem wirtschaftlichen und gesellschaftlichen Umfeld maßgeblich fördert.

Diese Evaluation hat des Weiteren gezeigt, dass der Aufbau dieser Netzwerke auch langfristig tragfähig ist. Viele der privaten Förderer unterstützen das Deutschlandstipendium nämlich über einen längeren Zeitraum hinweg: 86 Prozent der einmal gewonnen Förderer wollen sich auch künftig für das Deutschlandstipendium engagieren. Dies spricht für gelebte und gelungene Netzwerkarbeit und einen nachhalti- gen Nutzen des Programmes für Studierende, Hochschulen und Förderer.

\section{Finanzielle und ideelle Förderung}

Neben dem $B A f o ̈ G$, Bildungsdarlehen und den Stipendien der 13 vom $B M B F$ geförderten Begabtenförderungswerke hat sich das Deutschlandstipendium dank privatem Engagement als weitere Säule der Studienfinanzierung etabliert. Studierende können sich nun für eine Hochschule mit einer möglichst reichen und lebendigen Stipendien- und Vernetzungskultur entscheiden, wodurch finanzielle Hürden bei der Aufnahme und Durchführung eines Studiums verringert werden. Im Unterschied zu anderen Arten der Studienfinanzierung handelt es sich beim Deutschlandstipendium um eine nicht rückzahlungspflichtige Förderung, die mit dem Bezug von BAföG kombiniert werden kann.

Und es sind nicht die finanziellen Anreize allein, die Studierende zur Bewerbung um ein Deutschlandstipendium bewegen. Seit Bestehen des Deutschlandstipendiums sind die so genannten ideellen Fördermöglichkeiten immer wichtiger und vielschichtiger geworden. Diese können im Zusammenspiel der Hochschulen mit den Förderern oder auch aus Initiativen der Studierenden entstehen. Nachdem sich die Stipendiaten und Förderer auf Veranstaltungen wie der Stipendienvergabefeier näher kennen gelernt haben, können Förderer "ihren“ Stipendiaten beispielsweise unverbindliche Angebote in Form von Betriebs- und Werksführungen, Mentoringprogrammen, Workshops, Praktika oder anderen Austauschmöglichkeiten unterbreiten. Auch können die Kontakte als Möglichkeit zum Verfassen von Seminar- oder Abschlussarbeiten, beispielsweise mit Unternehmensbezug, genutzt werden. Und schließlich etablieren auch die Stipendiaten selbst eigene ideelle Austauschplattformen in Form von Vortragsreihen, Science Slams, Exkursionen, Stammtischen oder Projektgruppen, häufig flankiert durch einen regen Austausch in den Social MediaKanälen.

Neben der Bewertung von bislang erbrachten Leistungen in Studium und Schule begünstigt soziales Engagement, beispielsweise in Vereinen, der Politik, dem Umweltschutz oder Kultureinrichtungen, den Bewerbungserfolg. Dies gewährleistet, dass nicht allein die Noten eines Bewerbers für die Vergabe eines Stipendiums ausschlaggebend sind, sondern auch sein gesellschaftliches Verantwortungsgefühl und seine Bereitschaft, hier mitgestalten zu wollen. Ein im Jahr 2015 vom Stifterverband durchgeführter Wettbewerb mit dem Titel "Macht was draus!“ hat einen vertieften Einblick in das vielschichtige gesellschaftliche Engagement der Stipendiaten erlaubt und aus rund 80 Einreichungen zehn besonders überzeugende Initiativen mit insgesamt 30.000 Euro gefördert. Auch die im Jahr 2018 erstmalig 
durchgeführte "Sommerakademie Deutschlandstipendium" hat das Thema Engagement in den Fokus gerückt. Näheres dazu unter www.servicezentrum-deutschlandstipendium. de.

Und schließlich wird als drittes Förderkriterium auch die erfolgreiche Bewältigung von schwierigen sozialen, persönlichen oder familiären Bedingungen, beispielsweise eine Zuwanderergeschichte, Erlebnisse aus Flucht oder Vertreibung, die Erziehung von Kindern oder die Pflege und Versorgung von Angehörigen bei der Auswahl der Stipendiaten berücksichtigt. Dies soll dazu beitragen, die gesamte Persönlichkeit eines Bewerbers bestmöglich einschätzen und bewerten zu können.

Bezüglich der Sozialstruktur der Stipendiaten haben die erwähnte Evaluation und Begleitforschung des Deutschlandstipendiums gezeigt, dass das Deutschlandstipendium in dieser Hinsicht sehr ausgewogen ist bzw. der Zusammensetzung der Gesamtstudierendenschaft weitgehend entspricht (gemäß den Zahlen der Sozialerhebung des Deutschen Studentenwerks). Die Ergebnisse zeigen beispielsweise, dass der Anteil der Nicht-Akademikerkinder unter den Deutschlandstipendiaten mit 50 Prozent genauso hoch ist wie bei allen Studierenden. Die BAföG-Quote ist unter den Deutschlandstipendiaten mit 25 Prozent sogar höher als bei allen Studierenden (rund 20 Prozent) und auch der Anteil der Deutschlandstipendiaten mit Migrationshintergrund übertrifft den Anteil der Studierenden mit Migrationshintergrund bei allen Studierenden um 5 Prozentpunkte (28 Prozent gegenüber 23 Prozent).

\section{Gestaltung durch die Hochschulen}

An 90 Prozent der staatlichen Hochschulen werden Deutschlandstipendien vergeben. Sie tragen wie bereits erwähnt den größten Teil der operativen Arbeit bei der Umsetzung des Nationalen Stipendienprogrammes: Sie müssen die Förderer begeistern, langfristig binden und die Auswahlprozesse der Stipendiaten steuern. Daneben bietet ihnen das Deutschlandstipendium aber auch Chancen zur eigenen Profilbildung. Durch das Bekenntnis zur Unterstützung talentierter Studierender und den Ausbau der eigenen Stipendienkultur können sie ihre Studienbedingungen verbessern und die Attraktivität für talentierte junge Menschen erhöhen. Sie erhalten die Gelegenheit, das Thema Stipendienförderung selbstständig gestalten zu können und es nicht in externe Hände legen zu müssen.

Darüber hinaus bieten die Form der öffentlich-privaten Partnerschaft und der vergleichsweise geringe Förderbetrag von 150 Euro im Monat den Hochschulen eine gute Gelegenheit, sich mit ihrem gesellschaftlichen und wirtschaftlichen Umfeld zu vernetzen, beispielsweise mit den kleinen und mittelständischen Unternehmen der eigenen Region.
Viele Hochschulen haben auch so genannte Stipendienfonds eingerichtet, in die auch kleinere Einmalzahlungen getätigt werden können. Durch den Auf- und Ausbau der Kontakte zwischen einer Hochschule mit ihrem unmittelbaren Umfeld sind nicht selten langfristige Partnerschaften entstanden, die sich neben der Stipendienförderung auch auf Bereiche wie den Forschungs- und Wissenstransfer ausdehnen können.

Des Weiteren kann das Deutschlandstipendium für die Hochschulen ein guter Anlass sein, eigene und weiterführende Aktivitäten im Fundraising zu beginnen oder auszubauen. Langezeit herrschte bei vielen Hochschulen in Deutschland in diesem Punkt noch vornehme Zurückhaltung. Doch bietet sich gerade das Deutschlandstipendium als thematischer Einstieg und Anlass zur Etablierung geeigneter Strukturen an, bei denen auch von öffentlich finanzierten Unterstützungsangeboten Gebrauch gemacht werden kann. So erhalten die Hochschulen eine Pauschale vom Bund zur Akquisition privater Fördermittel. Die Praxis zeigt, dass insbesondere diejenigen Hochschulen beim Fundraising erfolgreich sind, bei denen sich die Leitungsebene zum Thema Fundraising offen bekennt und die Konzeption und Umsetzung von Fundraisingaktivitäten aktiv unterstützt. Um das Bewusstsein für die Bedeutung einer langfristigen Stipendien- und privaten Förderkultur weiter zu schärfen, sollte geprüft werden, ob dieses Thema auch in die jeweiligen Zielvereinbarungen zwischen den Hochschulen und den Bundesländern mit aufgenommen werden kann.

\section{Unternehmen als größte Förderergruppe}

Die Förderer des Deutschlandstipendiums lassen sich in drei große Gruppen einteilen: Unternehmen, Stiftungen und Privatpersonen. Die Evaluation des Deutschlandstipendiums hat ergeben, dass Unternehmen mit 65 Prozent die größte Fördergruppe darstellen, gefolgt von Stiftungen und Vereinen (20 Prozent) und Privatpersonen/Alumni (15 Prozent). Dabei ist das Deutschlandstipendium für alle Unternehmensbranchen gleichermaßen attraktiv: Eine hohe Beteiligung findet sich beispielsweise in den Bereichen Banken, Versicherungen, IT, Chemie, Pharma, Maschinenbau sowie Kfz-Produktion und -Zulieferung. Knapp 70 Prozent der fördernden Unternehmen haben mehr als 250 Beschäftigte und erwirtschaften über 50 Mio. Euro Jahresumsatz. Potential besteht daher noch bei der Einbindung von kleinen und mittleren Unternehmen (KMU). Gemessen an ihrem Anteil in der Gesamtwirtschaft sind die KMU unterrepräsentiert und sollten künftig durch weitere (Werbe-)Maßnahmen noch verstärkt eingebunden werden.

Für fördernde Unternehmen ist das Deutschlandstipendium in vielerlei Hinsicht attraktiv. Es gibt ihnen die Möglich- 
keit, sich als "Corporate Citizen" aktiv im Bildungsbereich zu engagieren und durch die direkte Unterstützung junger Menschen gesellschaftliche Verantwortung zu übernehmen. Auch verhindern sie durch ihr Engagement das Abwandern der jungen Talente aus ihrer Region. Daneben können sie von weiteren Vorteilen profitieren, die sich unmittelbar auf ihren Unternehmenserfolg auswirken, beispielsweise aus dem Bereich Personalgewinnung. So können sie ihre Fördermittel ganz gezielt für Studierende aus einer bestimmten Fachrichtung einsetzen und beratend in den Auswahlgremien der Hochschulen teilnehmen. In der genannten Evaluation und Begleitforschung führten die Förderer des Weiteren auch noch Ziele wie die finanzielle Entlastung von Studierenden, den Ausbau von Hochschulkooperationen sowie das Setzen von Anreizen für akademische Spitzenleistungen an.

In einem nächsten Schritt können sie zu „ihrem“ Stipendiaten direkten Kontakt aufnehmen, beispielsweise auf der Vergabefeier der Stipendien, und ihm bei beidseitigem Interesse Praktika oder den Besuch von Informations- und Weiterbildungsveranstaltungen anbieten. Daneben können Unternehmen von unterschiedlichen Kommunikationsleistungen profitieren, indem sie das Stipendium beispielsweise nach dem Unternehmensnamen benennen („Deutschlandstipendium der Firma X an der Hochschule $\mathrm{Y}^{\prime \prime}$ ) und in Verbindung mit dem Logo des Deutschlandstipendiums in der eigenen Öffentlichkeitsarbeit verwenden. Auf diese Weise wird das Deutschlandstipendium zu einer Win-
Win-Win-Situation für Förderer, Studierende und Hochschulen. Den größten unmittelbaren Nutzen haben dabei freilich die Studierenden in Deutschland. Doch auch für Hochschulen und Förderer ist es ein geeignetes Instrument, in Fragen der Bildungsförderung näher zusammenzurücken und aus diesem zukunftsrelevanten Thema ein gesamtgesellschaftliches Anliegen zu machen.

\section{Fazit}

Im Verlauf der letzten acht Jahre hat das Deutschlandstipendium immer weiter an Fahrt und Dynamik gewonnen: Die Anzahl an Studierenden ist kontinuierlich gewachsen und neue Themen wie die ideelle Förderung und von den Stipendiaten selbst organisierte Engagementprojekte sind entstanden. Vieles davon wird auch von den Hochschulen begleitet und aktiv unterstützt. Immer mehr private Förderer erkennen die Vorteile des Deutschlandstipendiums und engagieren sich im Bereich der Bildungsförderung. Als Konsequenz nimmt der Vernetzungsgrad der Hochschulen mit ihrem gesellschaftlichen und wirtschaftlichen Umfeld stetig zu. Auf diese Weise, und das ist die Hauptsache, werden auch in den kommenden Jahren immer mehr talentierte und sozial engagierte Studierende von diesem Fördermodell profitieren können und, wenn sie selbst ins Berufsleben starten, vielleicht ihrerseits zu Förderern des Deutschlandstipendiums werden. Auch hierfür gibt es bereits erste vielversprechende Beispiele. 